\title{
Understanding and Improving Deep Neural Network for Activity
}

\author{
Recognition ${ }^{\dagger}$
}

\author{
Li Xue, Si Xiandong ${ }^{\ddagger}$, Nie Lanshun ${ }^{\S}$, Li Jiazhen, Ding Renjie, Zhan Dechen, Chu Dianhui** \\ \{lixuecs@hit.edu.cn,15776633420@163.com, nls@hit.edu.cn \}
}

School of Computer Science and Technology, Harbin Institute of Technology

\begin{abstract}
Activity recognition has become a popular research branch in the field of pervasive computing in recent years. A large number of experiments can be obtained that activity sensor-based data's characteristics in activity recognition is variety, volume, and velocity. Deep learning technology, together with its various models, is one of the most effective ways of working on activity data. Nevertheless, there is no clear understanding of why it performs so well or how to make it more effective. In order to solve this problem, first, we applied convolution neural network on Human Activity Recognition Using Smartphones Data Set. Second, we realized the visualization of the sensor-based activity's data features extracted from the neural network. Then we had in-depth analysis of the visualization of features, explored the relationship between activity and features, and analyzed how Neural Networks identify activity based on these features. After that, we extracted the significant features related to the activities and sent the features to the DNN-based fusion model, which improved the classification rate to $96.1 \%$. This is the first work to our knowledge that visualizes abstract sensor-based activity data features. Based on the results, the method proposed in the paper promises to realize the accurate classification of sensor-based activity recognition.
\end{abstract}

Keywords: human activity recognition, DNN, feature extraction, data feature visualization

\section{Introduction}

With the development of the Internet of Things and big data, human activity recognition (HAR) has attracted much attention as a branch of pervasive computing. Recently, many academic researchers have started focusing on work that proposes various kinds of new methods to improve recognition performance [1][2][3][4]. HAR can also be seen as a branch of pattern recognition. Moreover, industry professionals have put significant effort into developing friendly sensors and equipment for human use. HAR is studied to solve practical problems, improve the safety and comfort people's life in their homes and daily common activities, and combining with other professional fields such as medicine [5][6], health [7][8], and sports [9][10] is an inevitable trend. Of course, these achievements get approved.

In the field of activity recognition,convolution neural network $(\mathrm{CNN})$ as the most basic model of deep learning shows excellent performance. In [11], they propose an approach to automatically extract discriminative features for activity recognition. Specifically, CNN-based methods can capture local dependence and a signal's scale invariance. Chen, Y., \& Xue, Y constructed a CNN model and modified the convolution kernel to adapt the characteristics of tri-axial acceleration signals [12]. Nine types of sensor information are used to synthesize a signal image for 2D image recognition in [13]. The work in [14] and [15] combines sensor data and time information into a two-dimensional matrix form, which is similar to single-axis picture information, preserves sensor correlations and time series information. Human activity data, based on sensors with high dimension characteristics and data features, are difficult to extract using traditional statistical methods. CNN is suitable for extracting high dimensional data, and the automatic extraction of data features is more suitable for the situation in which the data features of a sensor are not obvious in the activity recognition. Another reason that CNN can extract complex activity recognition data features mainly depends on three important features: local

\footnotetext{
${ }^{\dagger}$ Supported by National Natural Science Foundation of China No.61772159, Science and technology Special Project of National Regional Innovation Center No.2017QYCX12, Natural Science Foundation of Shandong Province No.ZR2017MF026, and HIT.NSRIF.201703 Innovation Foundation(Key Cultivation Project) of Harbin Institute of Technology No.HIT.NSRIF.201703.

* The first two authors contributed equally to this work

${ }^{\S}$ Corresponding author

${ }^{* *}$ Corresponding author
} 
connection, weight sharing, and translation invariance [16]. Therefore, this paper uses CNN to extract high-level data features.

Although CNN performs well, it has some problems: 1) It is hard to understand the internal mechanism of neural network. How neural networks recognize activity based on sensor data? What is the relationship between sensor data and activities? What is the impact of features extracted from the neural network on classification accuracy or recognition results of activities? How to improve the classification accuracy? [17], [18], [19], [20], [21]. 2) In some datasets, the accuracy of classifying activity applied neural networks isnot high enough and there is room for improvement, but the accuracy cannot be improved by the conventional methods such as tuning. At the same time, in some datasets, the classification accuracy of different activities is imbalanced [22] [23] [24]. Moreover, the accuracy of classifying some activities is relatively high while the accuracy on other activities is relatively low. These activities of low-accuracy classification become a factor that restricts improvement of accuracy of the whole datasets [25] [26]. So how to improve the recognition accuracy of low-accuracy activities?

To solve the problems above, we should try to understand the internal mechanism of neural network and the impact of sensor data and features on the classification results. Therefore, we visualized the features based on activity data. Currently, most researchers do not clearly know what the data features are extracted from the deep learning model and few publications on data feature visualization exist. In image processing, insight was given into the function of intermediate feature layers and the operation of classifiers [27]. They also perform an ablation study to discover the performance contributions of different model layers. The work in [28] makes it possible to learn about multiple layers of representation and we show models with four layers. According to the activated feature map in the visual convolution neural network, and the signal intensity of RSSI data, Liu, X., Liu, L., analyzed the important characteristics of the extraction phase in the execution stage of the emergency room [29]. Although CNN can extract high-level features of sensors well, the meaning of feature representation as extracted by the convolution kernel remains unclear, so it is necessary to analyze by visualization. Moreover, the past work did not use visual results to affect the classification accuracy. Therefore, this paper will visualize data features extracted from CNN and in this way we could know what data will have an obvious effect on the activity, in accordance with the results of visualization; we will extract significant data and send new data into the DNN-based fusion mode. Finally, the experiments show that the classification accuracy rate increased to $96.1 \%$.

In the paper, we presented visualizing and understanding $\mathrm{CNN}$-based activity recognition. In summary, this paper makes three contributions. First, we visualized abstract sensor-based activity data features. In-depth analysis of the relationship between sensor data and activities and the impact of features extracted from the neural network on classification results. The extracted features in the deep learning model are no longer a black box and help us to understand the mechanics inside neural networks. It also helps to understand activity and sensor data. Second, we proposed a DNN-based fusion model to improve the accuracy of classification, especially to improve the accuracy of these activities classified in low-accuracy. Third, we presented a promising method to solve the problem that deal with activities classified in low-accuracy. It provides ideas for further study in this field.

\section{Visualizing and Understanding CNN on dataset HARUSP}

The overall flow of experiments is: Train CNN to generate data features; Visualize features to generate feature graph; Analyze and understand features to get relationships between activities and features; Improve the performance of classification by the fusion Model. As shown in Figure 1.

\subsection{Dataset}

This paper is just for providing ideas to readers, so we have only carried out experiments on one dataset. We selected a common dataset in the field of HAR: HARUSP, and its basic information is shown in Table 1.

The following points require explanation:

1) The data matrix of this dataset is composed of nine columns, and they represent the body acceleration $x, y, z$ axes, gyroscope $x, y, z$ axes, and total acceleration $x, y, z$ axes, respectively.

2) The values of the $0^{\text {th }}, 1^{\text {st }}$, and $2^{\text {nd }}$ columns are mainly affected by the displacement of the human 
body. The $3^{\text {rd }}, 4^{\text {th }}$, and $5^{\text {th }}$ columns are mainly influenced by the human body rotation, and the $6^{\text {th }}$, $7^{\text {th }}$, and $8^{\text {th }}$ columns mainly represent the total acceleration. It is assumed that when the smartphone is stationary, it is also under gravitational acceleration of value $1 g$, so the acceleration data obtained from the sensor contains the gravity acceleration and the human body's acceleration as the total acceleration.

3) In Figure 2(a), we can know that the smartphone is tied around the waist. Figure2 (b) is the accelerometer's direction in the three axes and Figure (c) is the gyroscope's direction in the three axes.

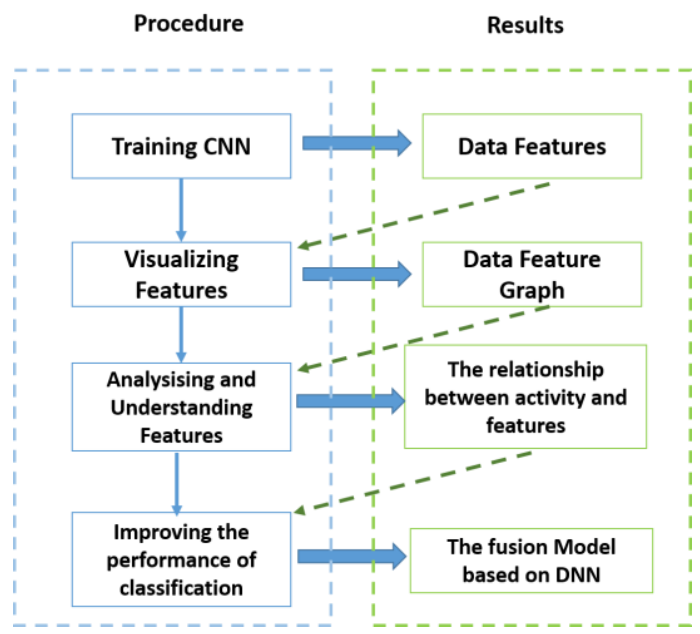

Figure 1.Experimental procedure

Table 1. The basic information of dataset

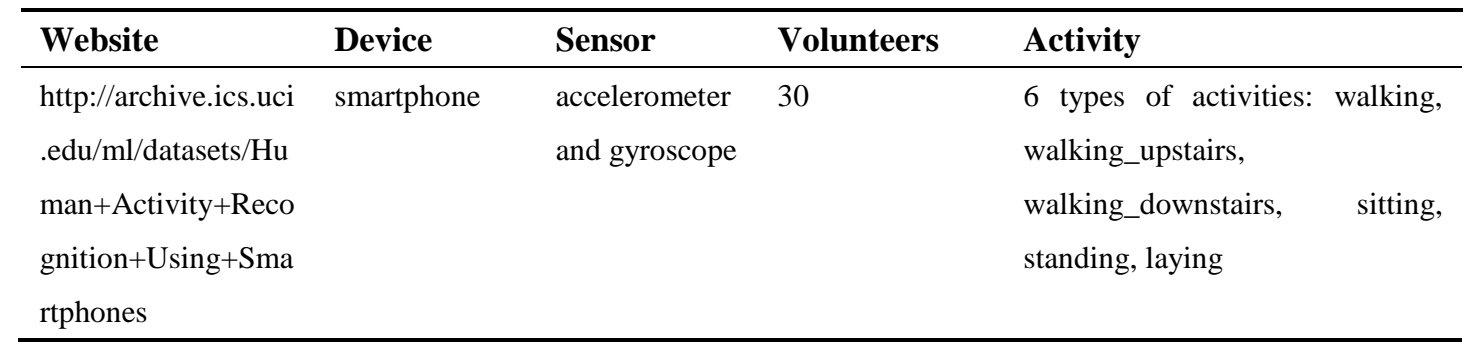

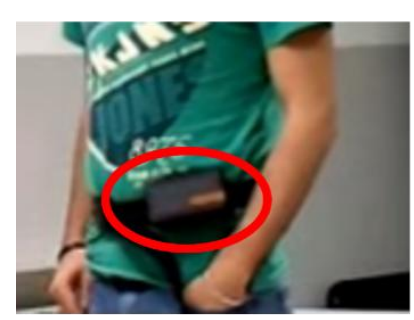

(a)

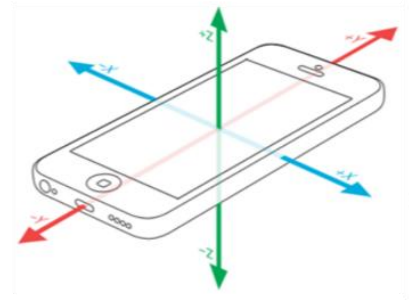

(b)

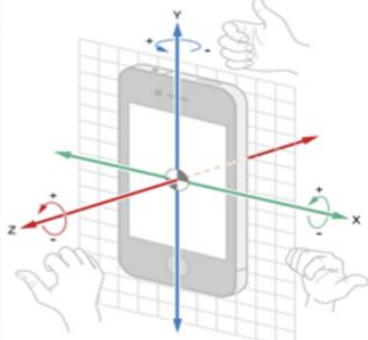

(c)

Figure 2. Placement map of smartphone and its sensors

\subsection{CNN-Based feature extraction on dataset HARUSP}

\subsubsection{CNN Model}

Figure3 shows the procedure of processing raw sensor data in CNN. First, the raw data is preprocessed and put into the CNN, 2D convolution is carried out, and then the ReLU activation function is used for nonlinear processing. After each convolution layer, we use the max pooling layer to further extract significant features from the sensor data.After high level data feature extraction in the convolution and pooling layer, adding dropout and batching normalization in the network structure can 
prevent over-fitting. The output of the convolution layer and pooling layer represents advanced features of the input sensor data.

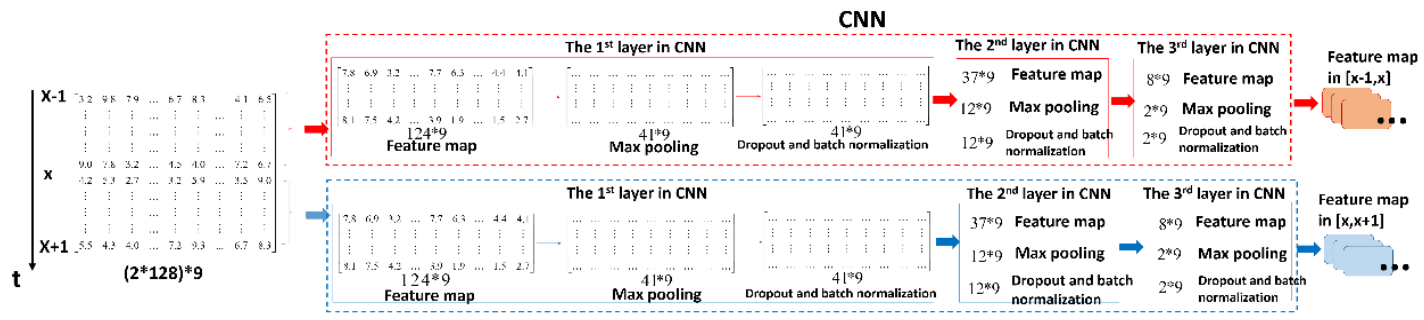

Figure 3. The procedure of processing raw sensor data in $\mathrm{CNN}$

Table 2 shows the basic parameters needed in CNN. It should be noted that these parameters are set based on experiential knowledge.

Table 2. The basic parameters needed in CNN

\begin{tabular}{lc}
\hline & HARUSP \\
\hline The size of input vector & $128 * 9$ \\
The number of kernel & 50 \\
Convolution kernel & $5 * 1$ \\
Pooling size & $3 * 1$ \\
The Probability dropout & 0.5 \\
Learning Rate & 0.01 \\
The number of iterations & 50 \\
The number of samples for each iteration & 32 \\
\hline
\end{tabular}

\subsubsection{Training CNN}

The process of training $\mathrm{CNN}$ requires the following steps:

1) Initialize all filters and parameters/weights with random numbers;

2) The network takes the sensor data window as input, performs forward propagation (including convolution, ReLU function, max pooling, and forward propagation of the full connection layer), and calculates each class' corresponding output probabilities. Suppose that the output probability currently is $[0.1,0.5,0.1,0.3]$; the weights of the first training sample are all random, so this output probability is also similar to the probability of random output.

3) Calculate the total error of the output layer. This paper uses the cross-entropy loss function:

$$
\mathrm{C}=-\frac{1}{\mathrm{~N}} \sum_{\mathrm{X}}[\mathrm{y} \ln \mathrm{a}+(1-\mathrm{y}) \ln (1-\mathrm{a})]
$$

$y$ is the actual output and $a$ is the output value of the neuron. When the real output $a$ is close to the expected output $y$, the cost function is close to 0 .

4) The backpropagation algorithm calculates errors relative to gradient of all weights and uses the gradient descent algorithm to update all weights and parameters in networks, thus minimizing the loss function.

When the sensor data is input again, the probability of the output may be $[0.1,0.1,0.7$, and 0.1$]$, which is closer to the goal of $[0,0,1$, and 0$]$. Therefore, the process of training CNN includes constantly updating weights and parameters.

\subsection{Visualizing and Understanding CNN-Based Activity Recognition on HARUSPDataset}

The following two points need to be explained:

1) We built two-dimensional feature graphs in which the longitudinal axis represents the time series in the time window; i.e., each row represents data at a time, and the horizontal axis represents the data of nine attributes collected by sensors. 
2) We performed the visualization of each type of activity layer-by-layer from a one-layer CNN to a five-layer CNN. According to this experiment, we can see the influence of the CNN layer on the feature extraction and each activity data feature graph in each layer is composed of one-time window selected randomly by the system. Then, we did three-layer CNN extraction feature visualization for each activity type. According to this experiment, we can see which data features can significantly influence the activity, and each activity data feature graph is composed of six-time windows that are selected randomly by the system.

\subsubsection{Visualization of activity data feature graph extracted layer-by-layer}

Different features are extracted from different numbers of layers in $\mathrm{CNN}$, to better understand the effect of different numbers of layers, we visualize the features extracted from one-layer to five-layer. Figure4 shows six kinds of activity data feature graphs extracted from CNN layer-by-layer. Overall, all six kinds of activity data feature graphs on the third layer are more obvious and more discriminative. Meanwhile, different activities have different main characteristics that facilitate the distinguishing of classifiers. Moreover, we do experiments about the CNN structure whose layers are from 1 to 5 on HARUSP. As you can see from Figure5, the accuracy and F1-score of the three-layer CNN structure are the best. Therefore, we study the features of the three-layer in depth.

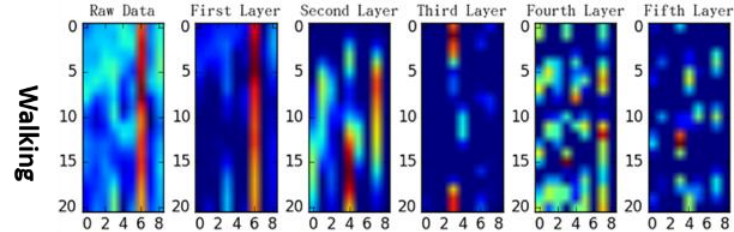

(a)
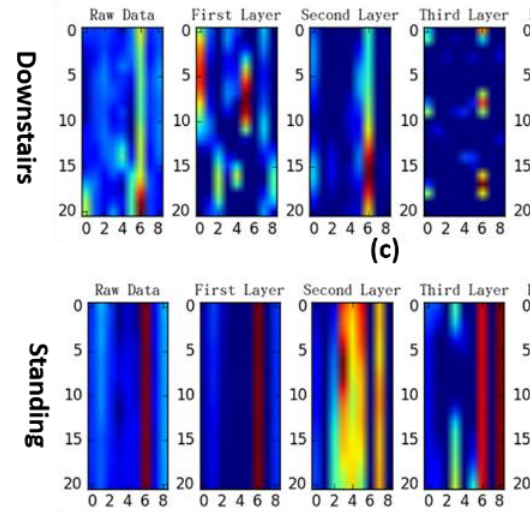

(e)
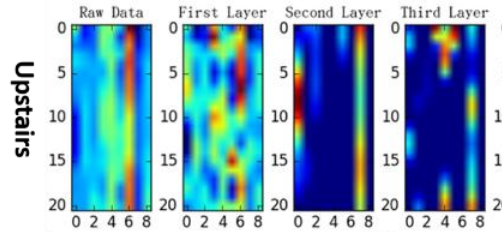

(b)
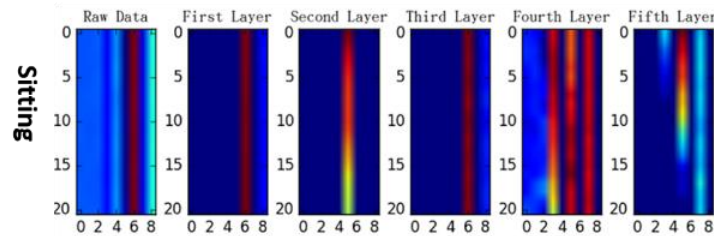

(d)
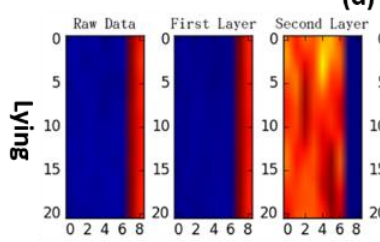

(f)

Figure 4.Six activity data feature graphs extracted layer-by-layer from CNN

\subsubsection{Visualization of activity data feature graph extracted from three-layer CNN}

We studied in detail the experiment scenarios and experiment setup for this dataset and analyzed the impact of activity on sensor data according to the experimentally recorded video to help us understand the relationship between features and activity. Figure6 shows the feature graphs of the six types of activity after convolution of three-layer CNN. Table 4 shows the detailed description of Figure6.

According to the above detailed analysis, each activity has its own significant features columns. we can see these columns have the greatest impact on the recognition of activities. (see Table 3 ) .

Table 3.The relationship between column and activity

\begin{tabular}{lllllll}
\hline & Walking & Upstairs & Downstairs & Sitting & Standing & Lying \\
\hline Features columns & 3,4 & 0,7 & 0,6 & 6 & 3,6 & 7,8 \\
\hline
\end{tabular}




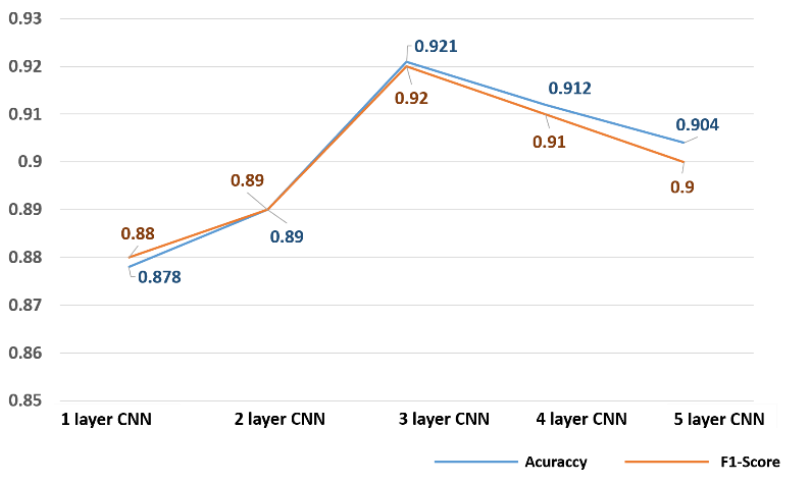

Figure 5.The performance of the CNN structure with 1-layer to 5-layer on HARUSP
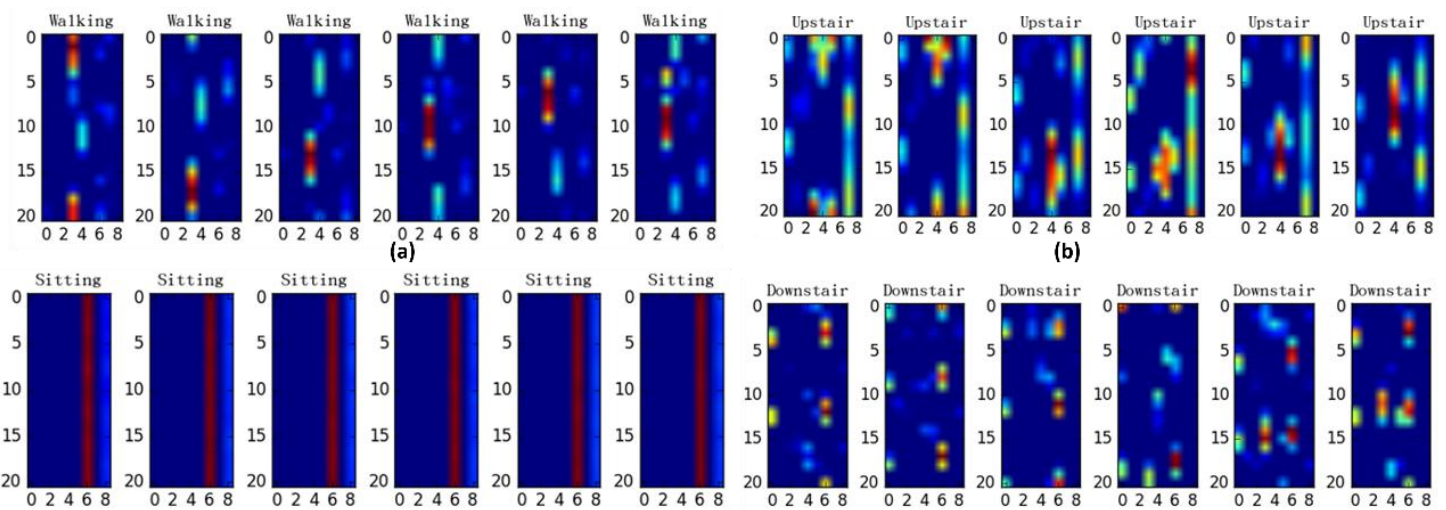

(d)
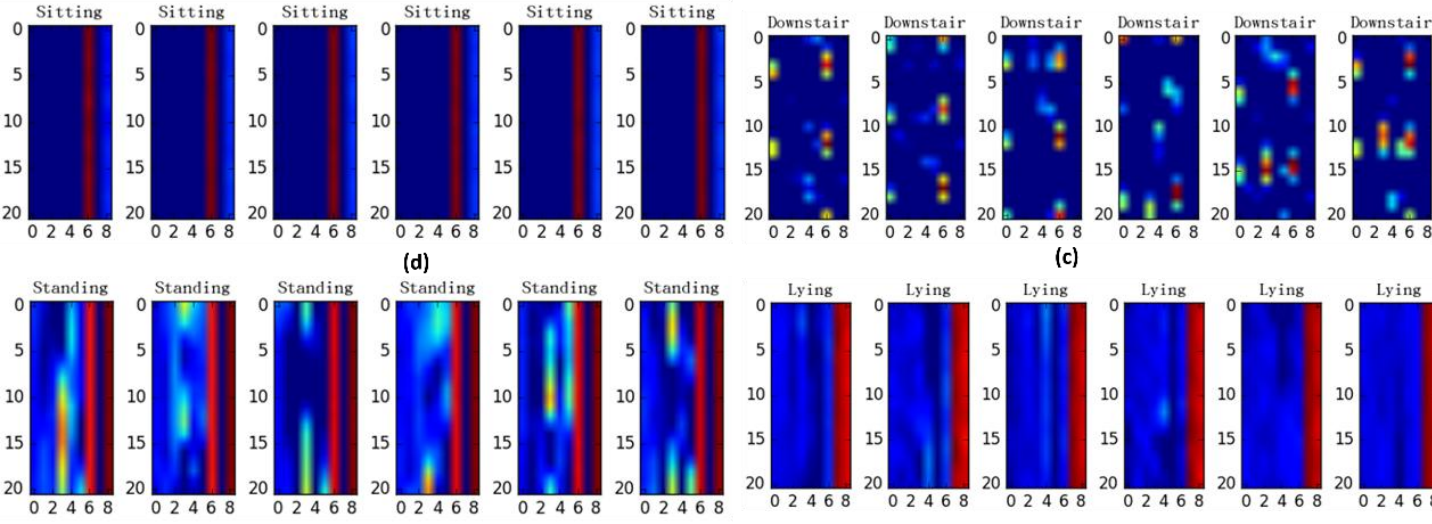

(e)
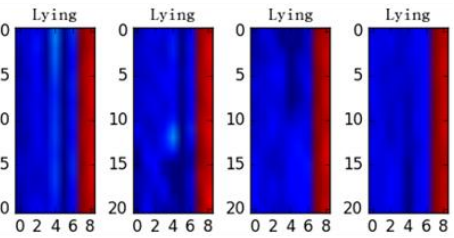

(f)

Figure 6. Six activity data feature graphs extracted from a three-layer CNN

Table 4. The detail description of Figure6

\begin{tabular}{|c|c|c|c|}
\hline activity & Analysis combined with the real-life scenarios & $\begin{array}{l}\text { The number of columns } \\
\text { of the significant features } \\
\text { in the feature map }\end{array}$ & $\begin{array}{l}\text { Whether the actual } \\
\text { analysis and the } \\
\text { visualization result } \\
\text { are consistent }\end{array}$ \\
\hline walking & $\begin{array}{l}\text { People walk no longer in a straight } \\
\text { line--->Affect the x-axis of Figure } 2 \text { (c); Phone } \\
\text { will rotate slightly around y axes--->Affect the } \\
\text { y-axis of Figure } 2 \text { (c) }\end{array}$ & $\begin{array}{l}\text { the } 3 \text { th column in Figure } \\
6 \text { (a); } \\
\text { the } 4 \text { th column in Figure } \\
6 \text { (a) }\end{array}$ & consistent \\
\hline Upstair & $\begin{array}{l}\text { The body's displacement will happen in the } \\
\text { upper and lower range---> Affect the x-axis of } \\
\text { Figure } 2 \text { (b); The weight of gravity acceleration } \\
\text { when going upstair--->Affect the y-axis of } \\
\text { Figure } 2 \text { (b) }\end{array}$ & $\begin{array}{l}\text { the 0th column in Figure } \\
6 \text { (b); } \\
\text { the } 7 \text { th column in Figure } \\
6 \text { (b) }\end{array}$ & consistent \\
\hline Downstair & $\begin{array}{l}\text { The body's displacement will happen in the } \\
\text { upper and lower range---> Affect the x-axis of } \\
\text { Figure } 2 \text { (b); The weight of gravity acceleration }\end{array}$ & $\begin{array}{l}\text { the 0th column in Figure } \\
6 \text { (c); } \\
\text { the } 6 \text { th column in Figure }\end{array}$ & consistent \\
\hline
\end{tabular}




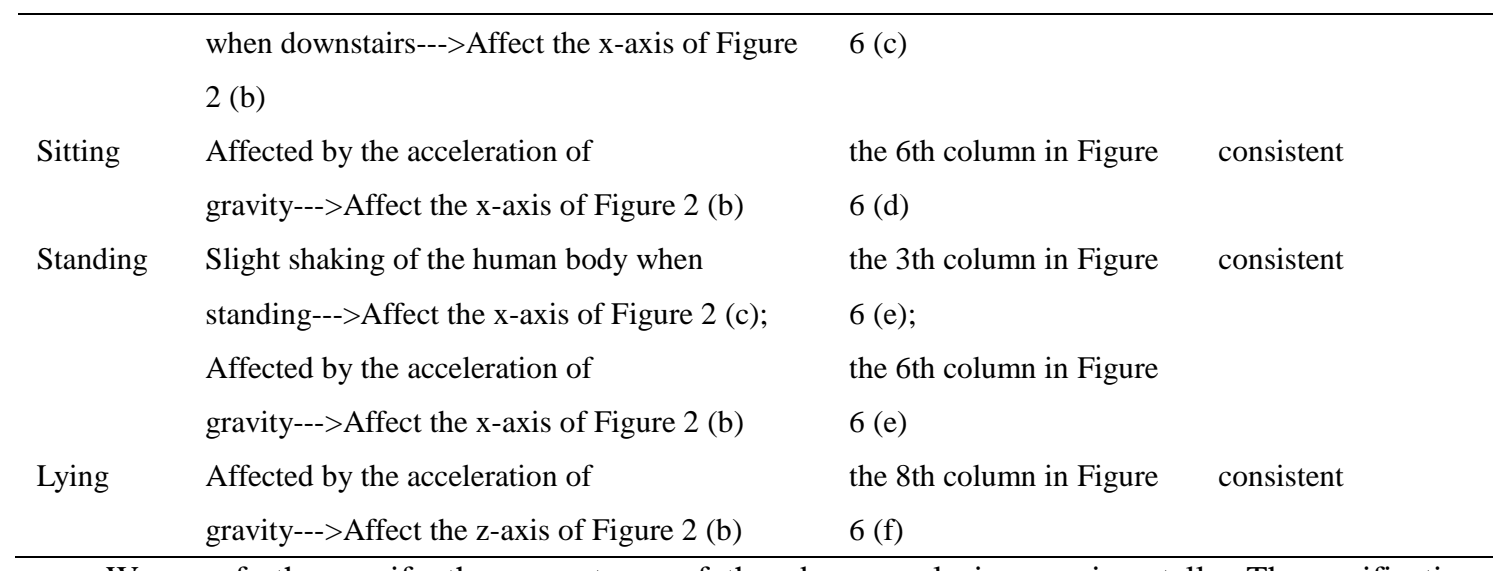

We can further verify the correctness of the above analysis experimentally. The verification method is occlusion[27]; the specific practice is covering a column of data (setting the value as 0), then checking the influence on the effect of classification. Finally, we determine which data has an impact on various types of activity. We used the above method to verify each of the activities on the HARUSP dataset, as shown in Table 5.Take Sitting as an example, when covering the 0th columns, 99\% of the samples will still be classified into walking, indicating that the 0th column is not the main feature of walking activities. When covering the 6th column, only $9 \%$ of samples will be classified as walking, indicating that the sixth columns are the main characteristics of walking activities. The same way as other activities are verified, we mark the column that each activity involves as yellow. As you can see, the results of Table 3 are essentially consistent with those of Table 5.

Table 5. The experiment result of occlusion method

\begin{tabular}{lllllll}
\hline & Walking & Upstairs & Downstairs & Sitting & Standing & Lying \\
\hline Sample number & 477 & 435 & 364 & 411 & 475 & 535 \\
The $\mathbf{0}^{\text {th }}$ Column & $99 \%$ & $28 \%$ & $4 \%$ & $99 \%$ & $99 \%$ & $100 \%$ \\
The $1^{\text {st }}$ Column & $98 \%$ & $98 \%$ & $100 \%$ & $96 \%$ & $100 \%$ & $100 \%$ \\
The $2^{\text {nd }}$ Column & $99 \%$ & $96 \%$ & $100 \%$ & $95 \%$ & $100 \%$ & $100 \%$ \\
The $3^{\text {rd }}$ Column & $19 \%$ & $84 \%$ & $76 \%$ & $100 \%$ & $39 \%$ & $99 \%$ \\
The $4^{\text {th }}$ Column & $54 \%$ & $96 \%$ & $89 \%$ & $83 \%$ & $100 \%$ & $100 \%$ \\
The $5^{\text {th }}$ Column & $60 \%$ & $97 \%$ & $94 \%$ & $97 \%$ & $99 \%$ & $100 \%$ \\
The $6^{\text {th }}$ Column & $94 \%$ & $97 \%$ & $95 \%$ & $9 \%$ & $52 \%$ & $100 \%$ \\
The $7^{\text {th }}$ Column & $96 \%$ & $46 \%$ & $100 \%$ & $90 \%$ & $86 \%$ & $89 \%$ \\
The $\mathbf{8}^{\text {th }}$ Column & $99 \%$ & $96 \%$ & $96 \%$ & $96 \%$ & $98 \%$ & $92 \%$ \\
\hline
\end{tabular}

In this chapter we visualized CNN-based activity recognition on the HARUSP dataset. Through visualization, the sensor data becomes intuitive. Then we get a sufficient understanding of activities, sensor data, and the mechanisms inside neural networks. Because of the different types of activities, the effects of activities on the sensors are different, and the collected sensor data has different characteristics. Neural networks extract different features based on these sensor data. For each activity, some of the features have no effect on the recognition of the activity while other features have a great impact on it, so different activities have their own significant features. At the same time, the neural networks mainly identify activities based on these significant features.

In the dataset, misclassification was mainly due to standing and sitting. The main reason is that these two types of activities have the same features (the 6th column), representing the effect of the acceleration of gravity. The difference is that when the human body stands, there is a slight rotation, the standing activity has the features of the third column, but the features is not obvious because of the small rotation of the human body. As a result, the classification error rate of the two activities is relatively high. After understanding of the visualization features, we proposed a fusion model to improve the accuracy.

\section{The DNN-based fusion model and Results}

Based on the above visualization, we clearly understand the relationship between activity and 
sensor data, and we find that each activity has corresponding significant features. Activity recognition is mainly based on the significant features, while other features have little effect on the classification. So we extracted the significant features related to the activity and used these features to train the model. Because of the use of the significant features and the exclusion of extraneous features, the model can better recognize difficult-to-distinguish activities and improve the recognition accuracy of all activities. Therefore, in the experiment, we use the main features to train two models, and use all features to train one model, and then use the voting mechanism to fuse the classification results of these three models to improve the recognition accuracy.

According to the above analysis, the two activities that are difficult to distinguish are sitting and standing. The feature columns related to these two activities are the $3 \mathrm{rd}$, 4th, $5 \mathrm{th}, 6 \mathrm{th}$, $7 \mathrm{th}$, and $8 \mathrm{th}$, of which the $3 r d, 4 t h, 6 t h$, and 7 th columns are more influential. Therefore, in the experiment, we trained three models:1) used all the data in the dataset; 2) extracted the $3 \mathrm{rd}$, 4th, 5th, 6th, $7 \mathrm{th}$, and 8th columns of data; 3 ) extracted the $3 \mathrm{rd}$, 4th, $6 \mathrm{th}$, and 7 th columns of data for training, and then used the voting mechanism to fuse the results of the three models.

Figure7 shows the training procedure of our model. During the training process, the training data were copied into three copies, they are respectively Train Data1, Train Data2, and Train Data3, respectively. Train Datal is sent directly into the Model-M for training, in which the Model-M structure is three-layer CNN and one-layer LSTM; CNN is responsible for extracting high-level activity data features, LSTM is mainly used to describe the correlation between the activity data time window, the final classification in the Softmax layer. The data of the 3rd, 4th, 5th, 6th, 7th, and 8th columns in the Train Data2 were sent into the Model-ml for training, in which the structure of Model-m1 was the same as that of Model-M. The data of $3 \mathrm{rd}, 4 \mathrm{th}, 6 \mathrm{th}$, and $7 \mathrm{th}$ columns in the Train Data3 are sent into the Model-m2 for training, in which the structure of Model-m2 is the same as that of Model-M.

Figure8 shows our model's testing procedure. In the process of testing, each piece of data in the test data is copied into Test Data1, Test Data2, and Test Data3. Test Datal is sent into Model-M to get the test results of Classification results-M, Test Data2 is sent into Model-m1 to get the test results of Classification results- $m 1$, and Test Data3 is sent into Model-m2 to get the test results of Classification results- $m 2$. The three classification results then use the voting mechanism to produce the final classification result. If there are two or three consistent results, the final classification results are the consistent results. If the three classification results are not the same, then the final classification results are Classification results- $M$.

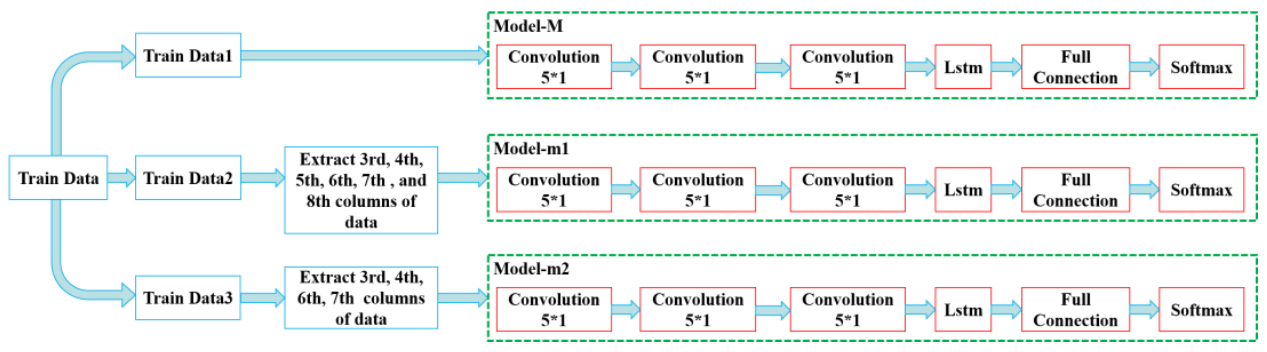

Figure 7.Training procedure of the DNN-based fusion model

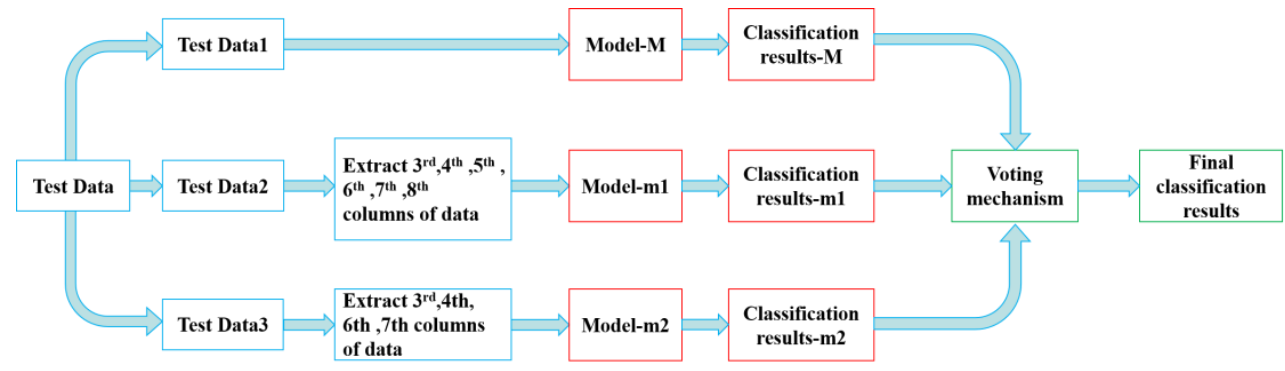

Figure 8. Classifying procedure of the DNN-based fusion model

The results are shown inTable 6.

Table 6.Comparision of performance of several models

\begin{tabular}{lccccccc}
\hline & CNN & LSTM & Paper[34] & Paper[35] & Paper[36 ] & CNN-LSTM & Our method \\
\hline Accuracy & 0.919 & 0.892 & 0.89 & 0.893 & 0.962 & 0.951 & $\mathbf{0 . 9 6 1}$ \\
F1-Score & 0.92 & 0.89 & n/a & n/a & n/a & 0.95 & $\mathbf{0 . 9 6}$ \\
\hline
\end{tabular}


Next, we compared the confusion matrix of the two methods. This paper focuses on the deep learning model, so we selected a high recognition rate method (CNN-LSTM) for comparison with our method. The confusion matrixes are listed in Figure9; the activities in a row represent predicted activities and the activities in a column are real activities. According to the comparison, it is found that the activities our method improves upon compared to the ordinary CNN-LSTM are mainly the two activities of Sitting and Standing; this gave us great inspiration. We found that this method is effective at distinguishing undistinguishable activities. However, since this is just the result of manual extraction, we will propose a method of automatically extracting sensor-based activity data features in future work, so that the new features can improve the accuracy of activity recognition by enhancing the data model.

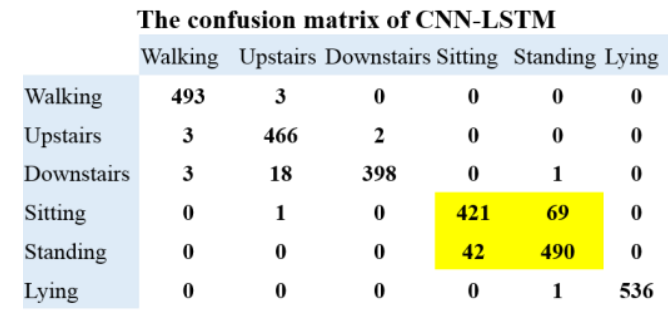

\begin{tabular}{l|ccccccc} 
& \multicolumn{7}{c}{ The confusion matrix of our method } \\
\cline { 2 - 5 } & Walking & \multicolumn{4}{l}{ Upstairs } & \multicolumn{4}{c}{ Downstairs } & Sitting & Standing & Lying \\
Walking & $\mathbf{4 8 7}$ & $\mathbf{6}$ & $\mathbf{3}$ & $\mathbf{0}$ & $\mathbf{0}$ & $\mathbf{0}$ \\
Upstairs & $\mathbf{1}$ & $\mathbf{4 6 8}$ & $\mathbf{2}$ & $\mathbf{0}$ & $\mathbf{0}$ & $\mathbf{0}$ \\
Downstairs & $\mathbf{0}$ & $\mathbf{1 3}$ & $\mathbf{4 0 7}$ & $\mathbf{0}$ & $\mathbf{0}$ & $\mathbf{0}$ \\
Sitting & $\mathbf{0}$ & $\mathbf{2}$ & $\mathbf{0}$ & $\mathbf{4 3 1}$ & $\mathbf{5 8}$ & $\mathbf{0}$ \\
Standing & $\mathbf{0}$ & $\mathbf{0}$ & $\mathbf{0}$ & $\mathbf{2 9}$ & $\mathbf{5 0 3}$ & $\mathbf{0}$ \\
Lying & $\mathbf{0}$ & $\mathbf{0}$ & $\mathbf{0}$ & $\mathbf{0}$ & $\mathbf{0}$ & $\mathbf{5 3 7}$
\end{tabular}

Figure 9. The two confusion matrixes

\section{Conclusion and Outlook}

This paper presented a novel idea for sensor-based activity recognition. We introduced the application of activity recognition in Deep Neural Network model and the visualization of data features in deep learning. Furthermore, the most important is that we visualize sensor-based activity data features extracted from $\mathrm{CNN}$ and the data features are abstract for a large number of researchers. This article is intended to provide a way of thinking for scholars, so we made an in-depth analysis of the visual feature maps on a single dataset HARUSP, which includes the visualization of activity data feature graph extracted from layer-by-layer and three-layer CNN. After the analysis, we manually extracted important data characteristics to our model, which have a greater impact on activities. We then verified the correctness of manual feature extraction from two aspects of theory and experiment. The classification accuracy was increased to $96.1 \%$. Experiments revealed that this paper can help solve the problems in Chapter 2.1. Therefore, it is verified by experimentation that our proposed idea is promising, which lays the groundwork for subsequent precision classification.

Through the in-depth analysis of the features, we have identified the relationships among activities, sensor data, and features. In addition, we analyzed how Deep Neural Network model recognize activities based on these features. The significant features mentioned in the experiment can be used not only in the method of the paper but also in other methods and ideas, which was greatly significant to the improvement of activity recognition. Through visualization, we have a deeper understanding of the mechanism inside the neural network. The ideas, findings and conclusions in the experiment are applicable not only to the field of activity recognition but also to other areas of deep learning.

However, in practice, the manual extraction of features is difficult to verify, particularly in the case of theoretical verification. It is necessary to know the location of sensor when each activity occurs. In future work, we will first present a method for extracting main features automatically. In addition, we will ensure the accuracy of the enhancement by considering presenting a fusion model that combines the main features with the original features to enhance the data.

\section{References}

[1] Jiang, Wenchao, and Zhaozheng Yin: Human Activity Recognition Using Wearable Sensors by Deep Convolutional Neural Networks. ACM multimedia . pp.1307-1310(2015)

[2] Ravi, D., Wong, C., Lo, B., \& Yang, G. Z: Deep learning for human activity recognition: A resource efficient implementation on low-power devices. Vol.3, pp.71-76. International Conference on Wearable and Implantable Body Sensor Networks (2016).

[3] Yao, Lina, et al. "Learning from less for better: semi-supervised activity recognition via shared structure discovery." ACM International Joint Conference on Pervasive and Ubiquitous Computing ACM. 
pp.13-24(2016).

[4] Chen, Kaixuan, et al. "Fullie and Wiselie: A Dual-Stream Recurrent Convolutional Attention Model for Activity Recognition." (2017).

[5] Nguyen, L. N., Rodriguezmartin, D., Catala, A., Perezlopez, C., Sama, A., \& Cavallaro, A. Basketball Activity Recognition using Wearable Inertial Measurement Units. international conference on human computer interaction. (2015).

[6] Blank, P., Schuldhaus, D., \& Eskofier, B. M. Sensor-based stroke detection and stroke type classification in table tennis. ACM International Symposium on Wearable Computers. pp.93-100(2015).

[7] Osmani, V., Balasubramaniam, S., \& Botvich, D.. Human activity recognition in pervasive health-care: Supporting efficient remote collaboration. Journal of Network and Computer Applications. pp.628-655(2008).

[8] Alshurafa, N., Xu, W., Liu, J. J., Huang, M., Mortazavi, B., Roberts, C. K., \& Sarrafzadeh, M.. Designing a Robust Activity Recognition Framework for Health and Exergaming Using Wearable Sensors. IEEE Journal of Biomedical and Health Informatics. pp.1636-1646(2014).

[9] Li, X., Yao, D., Pan, X., Johannaman, J., Yang, J. W., \& Webman, R., et al.. Activity recognition for medical teamwork based on passive RFID. IEEE International Conference on Rfid.(2016).

[10] Parlak Polatkan, S.. Object detection and activity recognition in dynamic medical settings using rfid. Dissertations \& Theses - Gradworks. (2013)

[11] Zeng, M., Nguyen, L.T., Yu, B., Mengshoel, O.J., Zhu, J., Wu, P., Zhang, J.,et al: Convolutional neural networks for human activity recognition using mobile sensors. Mobile Computing, Applications and Services (Mobi- CASE). pp.197-205(2014).

[12] Chen, Y., \& Xue, Y: A Deep Learning Approach to Human Activity Recognition Based on Single Accelerometer. IEEE International Conference on Systems, Man, and Cybernetics. pp.1488-1492(2016)

[13] Jiang, W., \& Yin, Z.: Human activity recognition using wearable sensors by deep convolutional neural networks. Proceedings of the 23rd ACM international conference on Multimedia.pp.1307-1310 (2015)

[14] Singh, M. S., Pondenkandath, V., Zhou, B., Lukowicz, P., \& Liwicki, M.. Transforming sensor data to the image domain for deep learning - an application to footstep detection(2017).

[15] Daniele Rav`1, Charence Wong, Benny Lo and Guang-Zhong Yang: Deep learning for human activity recognition: A resource e_cient implementation on low-power devices. Wearable and Implantable Body Sensor Networks(2016).

[16] Zeng, M., Le, T. N., Yu, B., Mengshoel, O. J., Zhu, J., \& Wu, P., et al.: Convolutional Neural Networks for Human Activity Recognition using Mobile Sensors. International Conference on Mobile Computing, Applications and Services . pp.197-205(2015).

[17] Ryoo, M. S., \& Matthies, L.. First-person activity recognition: feature, temporal structure, and prediction. International Journal of Computer Vision. pp.307-328 (2016).

[18] Hu, J. F., Zheng, W. S., Lai, J., \& Zhang, J.. Jointly learning heterogeneous features for RGB-D activity recognition. Computer Vision and Pattern Recognition.pp.5344-5352(2015).

[19] Lara, O. D., \& Labrador, M. A.. A survey on human activity recognition using wearable sensors. IEEE Communications Surveys \& Tutorials.pp.1192-1209 (2013).

[20] Ma, M., Fan, H., \& Kitani, K. M.. Going Deeper into First-Person Activity Recognition. IEEE Conference on Computer Vision and Pattern Recognition. IEEE Computer Society. pp.1894-1903(2016).

[21] Reyes-Ortiz, J. L., Oneto, L., Parra, X., \& Anguita, D.. Transition-aware human activity recognition using smartphones. Neurocomputing. pp.754-767(2016).

[22] Roggen, Daniel ,et al.Deep convolutional and LSTM recurrent neural networks for multimodal wearable activity recognition.Sensors (Switzerland)(2016).

[23] Yang, J.B.; Nguyen, M.N.; San, P.P.; Li, X.L.; Krishnaswamy, S. Deep Con-volutional Neural Networks OnMultichannel Time Series For Human Activity Rec-ognition. In Proceedings of the 24th International JointConference on Artificial Intelligence (IJCAI). pp. 3995-4001(2015).

[24] Zeng, M., Le, T. N., Yu, B., Mengshoel, O. J., Zhu, J., \& Wu, P., et al. Convolutional Neural Networks for Human Activity Recognition using Mobile Sensors. International Conference on Mobile Computing, Applications and Services (pp.197-205) (2015).

[25] Davide Anguita, Alessandro Ghio, Luca Oneto, Xavier Parra and Jorge L. Reyes-Ortiz. Human Activity Recognition on Smartphones using a Multiclass Hardware-Friendly Support Vector Machine. International Workshop of Ambient Assisted Living(2012).

[26] Davide Anguita, Alessandro Ghio, Luca Oneto, Xavier Parra, Jorge L. Reyes-Ortiz. Energy Efficient Smartphone-Based Activity Recognition using Fixed-Point Arithmetic. Journal of Universal Computer Science (2013).

[27] Zeiler, M. D., \& Fergus, R. Visualizing and understanding convolutional networks.pp.818-833 (2013).

[28] Zeiler, M. D., Taylor, G. W., \& Fergus, R.. Adaptive deconvolutional networks for mid and high level feature learning. International Conference on Computer Vision. pp.2018-2025(2011).

[29] Liu, X., Liu, L., Simske, S. J., \& Liu, J.: Human Daily Activity Recognition for Healthcare Using Wearable and Visual Sensing Data. IEEE International Conference on Healthcare Informatics. pp.24-31 (2016). 